\title{
Assessment of Stay Green Genotypes of Sunflower for Root Traits under Different Soil Moisture Regimes
}

\author{
Guruprasad Hiremath ${ }^{1 *}$ and Hajisaheb L. Nadaf ${ }^{2}$ \\ ${ }^{1}$ Depeartment of Genetics and Plant Breeding, University of Agricultural Sciences, \\ Dharwad, Karnataka, India \\ ${ }^{2}$ AICRP on Groundnut, MARS, University of Agricultural Sciences, \\ Dharwad-580005, Karnataka, India \\ *Corresponding author
}

\begin{tabular}{|c|c|}
\hline & A B S T R A C T \\
\hline & $\begin{array}{l}\text { The phenomenon of stay-green and root traits are most important in drought tolerance } \\
\text { breeding. The present investigation was conducted to identify the drought resistant stay- } \\
\text { green genotypes with desirable root traits which are essential to extract moisture from } \\
\text { deeper layers of the soil under drought conditions. Seven stay-green genotypes along with }\end{array}$ \\
\hline & $\begin{array}{l}\text { three non-staygreen checks were evaluated for root traits in PVP pipes with different soil } \\
\text { moisture regimes under Factorial RBD design with two replications. Moisture stress was }\end{array}$ \\
\hline Staygreen, Root: & imposed from R1-R2 stage in moderate stress and from R1-R8 stage in severe stress \\
\hline $\begin{array}{l}\text { Shoot ratio, Leaf area, } \\
\text { Variability. }\end{array}$ & $\begin{array}{l}\text { treatment. The analysis of variance revealed the significant differences among the } \\
\text { genotypes for all the root traits observed. The genotypes DSR } 14 \text { and DSR } 47 \text { have }\end{array}$ \\
\hline Article Info & exhibited higher mean root length $(102.50 \mathrm{~cm}$ each $)$, root to shoot ratio $(0.87$ and 1.00$)$, \\
\hline $\begin{array}{l}\text { Accepted: } \\
\text { 10 September } 2017 \\
\text { Available Online: } \\
10 \text { November } 2017\end{array}$ & $\begin{array}{l}\text { stress condition when compared to other genotypes and checks. The checks Morden, RHA } \\
6 \mathrm{D}-1 \text { and RHA } 95 \mathrm{C}-1 \text { recorded mean root length respectively of } 53 \mathrm{~cm}, 15.5 \mathrm{~cm} \text { and } 47 \\
\mathrm{~cm} \text { and root to shoot ratio of } 0.66,0.44 \text { and } 0.73 \text {, respectively under severe moisture stress } \\
\text { condition. The average seed yield per plant for these identified genotypes, DSR } 14 \text { (13.74 }\end{array}$ \\
\hline & $\begin{array}{l}\text { g) and DSR } 47(12.38 \mathrm{~g}) \text { was higher than that of the checks, Morden }(5.38 \mathrm{~g}) \text {, RHA } 6 \mathrm{D}- \\
1(2.75 \mathrm{~g}) \text { and RHA } 95 \mathrm{C}-1(3.55 \mathrm{~g}) \text { under severe moisture stress condition. Hence, these } \\
\text { genotypes can be used in further recombination breeding programme for drought } \\
\text { tolerance. }\end{array}$ \\
\hline
\end{tabular}

\section{Introduction}

Sunflower (Helianthus annuus L.) is one among the most important oil seed crops, ranking second in importance after soybean in the world. The crop belongs to Asteracael Compositae family and native to the temperate North America. Because of its moderate cultivation requirements and high oil quality, its acreage has been increased in both developed and developing countries
(Skoric, 1992). Its oil is called premium oil because of high percentage of polyunsaturated fatty acids (60\%); including oleic acid (16.2\%) and linoleic acid (72.5\%), which help to control cholesterol in blood (Satyabrata et al., 1988). Sunflower has been successfully cultivated over a widely scattered geographical area in the world. It is a highly cross pollinated crop which is adaptable to a 
wide range of agro climatic situations, having high yield potential, suitable for cultivation in all seasons due to its photo insensitivity nature and can fit well in various inter and sequence cropping systems.

Drought is the most limiting of all abiotic stresses and it affects well over one-third of the soils worldwide (Skoric, 2009). Productivity of sunflower is strongly depended on availability of water and greatest yield losses occur when water shortage occurs at flowering stage (Soorninia et al., 2012). Evidence indicated that sunflower is most sensitive to water deficit stress at critical stages, flowering and seed-maturity (Chimenti et al., 2002), and drought stress during vegetative phase, flowering period and seed filling period causes considerable decrease in yield and oil content of sunflower (Ali et al., 2009). The identification of suitable genotypes exhibiting tolerance to moisture stress as well as those showing least per cent reduction in their growth and yield attributes under stress is necessary for the improvement of productivity under rainfed environment. Breeding for resistance to drought stress is one among the main objectives of plant breeding programs.

The choice of selection strategy is critical to breeding for stress tolerance. Among the several morpho-physiological traits to be used as selection criteria under drought, the stay green nature and root traits of the crop acts as most appropriate. Stay green phenotypes have given rise to better yield under water-limited conditions in several crops such as sorghum, wheat, rice, cotton and maize (Harris et al., 2007; Arriola et al., 2012; Jordan et al., 2012; Rong et al., 2013). In sunflower also, albeit the infancy of utilization of stay-green phenomenon while breeding for drought tolerance, best practical results have been achieved. Stay green is characterized by the retention of green leaf area at crop maturity under water stress. Staygreen is used not only in breeding for drought resistance but also in breeding for resistance to Phomopsis (Skoric, 1992) and Macrophomina, a fungal disease whose development is promoted by stress conditions (Skoric, 2009).

In several agriculturally important crops it has been clearly understood that root system is an important trait for drought tolerance studies. One of the central targets of trait-based crop research in this century is to increase the efficiency of the crop's root system. The study on root traits and its relevance to drought is its infancy stage and only few reports are available on characterized variation on sunflower root system (Rauf and Sadqat, 2008). However, to understand properly functions of other root traits, its relations and to know which root trait exhibit more vital role for increasing crop productivity by sunflower breeders are so important issues to develop more productive and drought tolerant lines in their breeding programs under water stress conditions (Comas et al., 2013).

Hence, the present investigation was envisaged to understand the root biology of staygreen genotypes under different soil moisture regimes and to isolate the staygreen genotype having the desired root traits.

\section{Materials and Methods}

The experiment was conducted by planting the seven staygreen genotypes (DSR 14, DSR 20, DSR 40, DSR 47, DSR 51, DSR 56 and DSR 57) of sunflower along with three non staygreen checks (Morden, RHA 95C-1 and RHA 6D-1) in light gray polyvinyl chloride (PVC) pipes with the dimension of $170 \mathrm{~cm}$ long and $25 \mathrm{~cm}$ diameter filled with soil mixture comprised of black loam soil, sand and farmyard compost (50:30:20) (Geetha et al., 2012). Experiment was laid out in 
Factorial Random Block Design with irrigation as one factors (well watered, moderately stress and severe stress) and genotypes as other factor (ten genotypes) with two replications at botanical garden, Department of Genetics and Plant Breeding, University of Agriculture, Dharwad, Karnataka, India.

\section{Management of irrigation}

In the control or well watered, moderately stressed and severe stress conditions, the pipes/pots were irrigated at 8 days intervals until flowering initiates (40 days after sowing). In the well watered condition, the crop was irrigated throughout the crop growth period whereas in moderate stress treatment, irrigation was withhold from flower bud initiation stage (R-1/R-2) i.e., 40 days after sowing (DAS) to 60 DAS (Rauf and Sadqat, 2008) and in severe stress treatment, the irrigation was withhold from R1 stage to till the crop attains physiological maturity (R-8)/ crop harvested (R-9) to achieve low soil moisture during the whole reproductive growth phase. During imposition of stress, water stress condition was maintained as rain out shelter to protect from rain.

\section{Root sampling}

Harvesting of the individual plants in all three treatments for recording of root traits was done at 90 DAS sowing. Pipes at ground level were removed carefully and soaked in moisture for overnight to loosen the soil. The next day, roots were washed thoroughly and carefully using a sieve and fine jet of water to remove the adhered soil and debris. The cleaned roots were collected in poly bag for recording observations. Observations viz., root length $(\mathrm{cm})$, shoot length $(\mathrm{cm})$, root to shoot ratio, root spread $(\mathrm{cm})$, leaf area $\left(\mathrm{cm}^{2}\right)$, root dry weight $(\mathrm{g})$, shoot dry weight $(\mathrm{g})$, head dry weight $(\mathrm{g})$, total dry matter $(\mathrm{g})$, head diameter (g) and seed yield (g) per plant (Geetha et al., 2012) was recorded and statistical analysis was done using Windostat software version 9.2.

\section{Results and Discussion}

The analysis of variance revealed the significant differences among the genotypes for all the root traits (root length, shoot length, root to shoot ratio, root spread, root dry weight, shoot dry weight, head dry weight, and total dry matter), leaf area, head diameter and seed yield per plant observed under different soil moisture regimes. There was significant interaction between the genotypes and different regimes of soil moisture (well watered, moderate and severe stress) observed under the investigation (Table 1) for all traits studied under the investigation. The genotypes, DSR 14 followed by DSR 47 and DSR 51 showed higher root length both under severe moisture stress and well watered condition. While, the genotype, DSR 47 followed by DSR 51 recorded high root length $(114 \mathrm{~cm}$ and $95 \mathrm{~cm}$, respectively) under moderate moisture stress. However under severe water stress, the staygreen genotypes, DSR 40 followed by DSR 51, DSR 47 and DSR 14 have exhibited increased root length of $125 \%, 9.63 \%, 6.77$ $\%$ and $2.50 \%$, respectively over the root length of these genotypes under well watered condition. While other three staygreen genotypes (DSR 56, DSR 57 and DSR 20) and three non- staygreen genotypes (Morden, RHA 6D-1 and RHA 95C-1) showed higher reduction in root length under severe moisture stress (Table 2) as compared to well watered condition. Increase in root length is an adaptive mechanism for drought tolerant genotypes. Therefore higher value may be used for the discrimination between drought tolerant and susceptible genotypes. Rauf and Sadqat, (2008) stated that increase in root length occurred due to higher osmotic 
adjustment ability of drought genotypes. Under moderate moisture stress, the plants were irrigated after the period of moisture stress at critical stage (40-60 DAS). Genotypes with improvement in root system including root length and seed yield are said to be recovered from the moisture stress and can be selected as drought tolerant for their utilization in breeding programme.

The genotypes, DSR 14 and DSR 47 showed high root length (Fig. 1) with an average root length of $102.50 \mathrm{~cm}$ each under severe stress condition, as against 100 and $96 \mathrm{~cm}$, respectively under well watered condition. The same genotypes also showed higher root to shoot ratio (0.87 and 1.00 , respectively) and root spread $(15.50 \mathrm{~cm}$ and $18.50 \mathrm{~cm}$, respectively) under severe soil moisture stress condition when compared to other genotypes and checks screened under the study.

The checks like Morden, RHA 6D-1 and RHA 95C-1 recorded mean root length respectively of $53 \mathrm{~cm}, 15.5 \mathrm{~cm}$ and $47 \mathrm{~cm}$ and root to shoot ratio of $0.66,0.44$ and 0.73 , respectively under severe moisture stress condition. These results are in accordance with the results of Rauf and Sadqat (2007), Rauf and Sadqat (2008), Eshghi et al., (2011) and Geetha et al., (2012). The genotypes with longer roots would allow water extraction from deeper soil profiles and thus, it is expected that the plant will perform better under moisture stress and can be considered as drought tolerant. Rachidi et al., (1993) reported that sunflower genotypes having deep and extensive root system can extract water up to depth of $270 \mathrm{~cm}$.

The genotypes DSR 47 followed by Morden, DSR 20 and DSR 14 showed higher root spread with better lateral roots under moisture stress (Table 3). Increase in root length occurred at expense of lateral roots. Root dry weight and total dry matter was reduced respectively by $31.36 \%$ and $28.01 \%$ by severe moisture stress in comparison with control or well watered. All the genotypes showed in reduction of root dry weight under moisture stress except DSR 56 which in contrary showed increased root dry weight by 25.93 per cent (Table 3). Pekcan et al., 2016 in Turkey reported that root fresh and dry weights are most affected by the drought stress as compared to total plant fresh and dry weight.

Most of the genotypes showed declining trend in shoot, root and head dry weight along with total dry matter under severe and moderate moisture stress. Genotype DSR 40 exhibited increased total dry matter $(8.95 \%)$ under severe moisture stress over the control and the least reduction of total dry matter was exhibited by the genotypes Morden, DSR 51 and DSR 56 while the highest reduction was in the genotypes RHA 6D-1, DSR 57 and RHA 95C-1. With limited water availability, it results lessening leaf growth then leading to decrease relative dry matter portioning into the root and shoot/root ratio (Rauf and Sadaqat, 2007).

All the genotypes under study showed the repressed effect on leaf area by the moisture stress with least effect in the genotype DSR 40 and DSR 51(Table 4). Higher leaf area was observed in the genotype DSR 14 and DSR 47 under severe and moderate moisture stress conditions. The genotypes with high leaf area under moisture stress are desirable as they had higher leaf surface area for photosynthesis to occur. The genotype DSR 47 had higher leaf area $\left(208.80 \mathrm{~cm}^{2}\right)$ than DSR 14 under moderate moisture stress which indicated that leaf area was improved upon relived from moisture stress. These genotypes which can regain from the stress are found superior for cultivation drought affected areas. Highest reduction in leaf area was found in Morden followed by RHA 95C-1 and RHA 6D-1. 
Table.1 Analysis of variance for different morpho-physiological and root traits under different soil moisture regimes in sunflower

\begin{tabular}{|c|c|c|c|c|c|c|c|c|c|c|c|c|}
\hline $\begin{array}{l}\text { Source of } \\
\text { variation }\end{array}$ & $\begin{array}{l}\text { Degrees } \\
\text { of } \\
\text { Freedom }\end{array}$ & $\begin{array}{c}\text { Shoot } \\
\text { Length } \\
(\mathbf{c m})\end{array}$ & $\begin{array}{c}\text { Root } \\
\text { Length } \\
\text { (cm) }\end{array}$ & $\begin{array}{l}\text { Root: } \\
\text { Shoot } \\
\text { ratio }\end{array}$ & $\begin{array}{c}\text { Root } \\
\text { Spread } \\
\text { (cm) }\end{array}$ & $\begin{array}{c}\text { Dry } \\
\text { Root } \\
\text { Weight } \\
\text { (g) }\end{array}$ & $\begin{array}{c}\text { Dry } \\
\text { Shoot } \\
\text { Weight } \\
\text { (g) }\end{array}$ & $\begin{array}{c}\text { Dry } \\
\text { Head } \\
\text { Weight } \\
\text { (g) }\end{array}$ & $\begin{array}{l}\text { Total Dry } \\
\text { Matter (g) }\end{array}$ & $\begin{array}{l}\text { Leaf Area } \\
\left(\mathrm{cm}^{2}\right)\end{array}$ & $\begin{array}{c}\text { Head } \\
\text { Diameter } \\
(\mathbf{c m})\end{array}$ & $\begin{array}{c}\text { Seed } \\
\text { Yield per } \\
\text { Plant (g) }\end{array}$ \\
\hline Replication & 1 & 45.07 & 98.82 & 0.03 & 8.07 & 2.40 & 28.70 & 8.94 & 46.22 & 0.08 & 0.62 & 1.67 \\
\hline Treatments & 41 & $957.96 * *$ & $1847.92 * *$ & $0.15 * *$ & $22.50 * *$ & $31.02 * *$ & $439.17 * *$ & $52.55 * *$ & $939.18 * *$ & $4546.95 * *$ & $15.52 * *$ & $33.34 * *$ \\
\hline 1. Stress & 1 & $972.02 * *$ & $2718.47 * *$ & $0.39 * *$ & 9.15 & $81.78 * *$ & $286.15 * *$ & $289.79 * *$ & $1787.88 * *$ & $20474.76 * *$ & $73.24 * *$ & $166.01 * *$ \\
\hline 2. Genotypes & 20 & $2479.49 * *$ & $4266.89 * *$ & $0.24 * *$ & $53.51 * *$ & $68.53 * *$ & $840.35 * *$ & $69.91 * *$ & $1941.76 * *$ & $7062.48 * *$ & $27.86 * *$ & $60.07 * *$ \\
\hline 3. Interaction & 20 & $195.63 * *$ & $541.71 * *$ & $0.07 * *$ & $8.48 *$ & $6.62 * *$ & $255.58 * *$ & $17.51 * *$ & $343.59 * *$ & $1519.43 * *$ & $2.93 *$ & $5.24 * *$ \\
\hline Error & 41 & 77.86 & 49.30 & 0.01 & 3.31 & 1.03 & 12.36 & 1.91 & 15.67 & 320.37 & 1.12 & 1.97 \\
\hline
\end{tabular}

Table.2 Mean performance of shoot length, root length and root to shoot ratio under different soil moisture regimes in sunflower

\begin{tabular}{|c|c|c|c|c|c|c|c|c|c|c|c|c|c|c|c|}
\hline \multirow[b]{2}{*}{ Genotypes } & \multicolumn{5}{|c|}{ Shoot Length $(\mathrm{cm})$} & \multicolumn{5}{|c|}{ Root Length (cm) } & \multicolumn{5}{|c|}{ Root:Shoot ratio } \\
\hline & $\mathbf{S}$ & MS & NS & Mean & $\begin{array}{l}\text { Per cent } \\
\text { Reduction }\end{array}$ & $\mathbf{S}$ & MS & NS & Mean & $\begin{array}{l}\text { Per cent } \\
\text { Reduction }\end{array}$ & $\mathbf{S}$ & MS & NS & Mean & \begin{tabular}{|l|} 
Per cent \\
Reduction \\
\end{tabular} \\
\hline DSR 14 & 118.50 & 122.00 & 131.50 & 124.00 & 9.89 & 102.50 & 74.50 & 100.00 & 92.33 & -2.50 & 0.87 & 0.61 & 0.76 & 0.75 & $\begin{array}{r}-13.61 \\
\end{array}$ \\
\hline DSR 57 & 82.00 & 88.00 & 90.50 & 76.17 & 9.39 & 61.00 & 21.50 & 86.50 & 56.33 & 29.48 & 0.75 & 0.25 & 0.96 & 0.65 & 21.68 \\
\hline DSR 51 & 81.50 & 88.00 & 100.50 & 90.00 & 18.91 & 102.50 & 95.00 & 93.50 & 97.00 & -9.63 & 1.26 & 1.08 & 0.93 & 1.09 & -34.65 \\
\hline DSR 56 & 92.00 & 89.50 & 91.50 & 91.00 & -0.55 & 51.00 & 68.50 & 73.00 & 64.17 & 30.14 & 0.55 & 0.77 & 0.80 & 0.71 & 30.27 \\
\hline DSR 20 & 102.00 & 103.50 & 101.50 & 102.33 & -0.49 & 58.50 & 52.00 & 82.00 & 64.17 & 28.66 & 0.57 & 0.50 & 0.85 & 0.64 & 32.86 \\
\hline DSR 40 & 80.50 & 104.50 & 83.50 & 89.50 & 3.59 & 72.00 & 13.50 & 32.00 & 39.17 & -125.00 & 0.90 & 0.13 & 0.39 & 0.47 & -132.58 \\
\hline DSR 47 & 103.00 & 140.00 & 118.00 & 120.33 & 12.71 & 102.50 & 114.00 & 96.00 & 104.17 & -6.77 & 1.00 & 0.81 & 0.81 & 0.87 & -22.09 \\
\hline Morden & 80.50 & 70.00 & 85.50 & 78.67 & 5.85 & 53.00 & 47.00 & 86.50 & 62.17 & 38.73 & 0.66 & 0.67 & 1.02 & 0.79 & 35.12 \\
\hline RHA 6D-1 & 36.50 & 60.00 & 62.50 & 53.00 & 41.60 & 15.50 & 10.50 & 33.00 & 19.67 & 53.03 & 0.44 & 0.18 & 0.53 & 0.38 & 16.71 \\
\hline RHA 95C-1 & 64.50 & 85.00 & 105.50 & 85.00 & 38.86 & 47.00 & 26.00 & 71.00 & 48.00 & 33.80 & 0.73 & 0.30 & 0.68 & 0.57 & -7.31 \\
\hline Mean & 84.10 & 95.05 & 97.05 & 91.00 & & 66.55 & 52.25 & 75.35 & 64.72 & & 0.77 & 0.53 & 0.77 & 0.69 & \\
\hline \multicolumn{4}{|c|}{ CD at $5 \%$ for Stresses (S) } & 5.71 & & & & & 4.54 & & & & & 0.07 & \\
\hline \multicolumn{4}{|c|}{ CD at $5 \%$ for Genotypes G) } & 10.42 & & & & & 8.29 & & & & & 0.12 & \\
\hline \multicolumn{4}{|c|}{ CD at $5 \%$ for Stress $\times$ Genotype } & 18.05 & & & & & 14.36 & & & & & 0.22 & \\
\hline
\end{tabular}

Note: S- Severe stress; MS- Moderate stress; NS- Non-stress/Control/Well watered 
Table.3 Mean performance of root spread, shoot and root dry weight under different soil moisture regimes in sunflower

\begin{tabular}{|c|c|c|c|c|c|c|c|c|c|c|c|c|c|c|c|}
\hline \multirow[b]{2}{*}{ Genotypes } & \multicolumn{5}{|c|}{ Root spread (cm) } & \multicolumn{5}{|c|}{ Shoot Dry weight (g) } & \multicolumn{5}{|c|}{ Root Dry weight (g) } \\
\hline & $\mathbf{S}$ & MS & NS & Mean & $\begin{array}{l}\text { Per cent } \\
\text { Reduction }\end{array}$ & $\mathbf{S}$ & MS & NS & Mean & $\begin{array}{l}\text { Per cent } \\
\text { Reduction }\end{array}$ & $\mathbf{S}$ & MS & NS & Mean & $\begin{array}{l}\text { Per cent } \\
\text { Reduction }\end{array}$ \\
\hline DSR 14 & 15.50 & 15.00 & 18.00 & 16.17 & 13.89 & 32.75 & 28.25 & 55.25 & 38.75 & 40.72 & 12.25 & 9.75 & 18.00 & 13.33 & 31.94 \\
\hline DSR 57 & 11.00 & 11.50 & 12.50 & 11.67 & 12.00 & 16.00 & 11.50 & 32.00 & 19.83 & 50.00 & 4.25 & 4.75 & 9.25 & 6.08 & 54.05 \\
\hline DSR 51 & 14.00 & 16.50 & 14.00 & 14.83 & 0.00 & 24.00 & 28.75 & 22.50 & 25.08 & -6.67 & 5.25 & 5.25 & 8.75 & 6.42 & 40.00 \\
\hline DSR 56 & 14.50 & 14.00 & 11.00 & 13.17 & -31.82 & 36.00 & 22.50 & 43.50 & 34.00 & 17.24 & 8.50 & 5.75 & 6.75 & 7.00 & -25.93 \\
\hline DSR 20 & 16.50 & 16.00 & 19.00 & 17.17 & 13.16 & 26.75 & 16.75 & 43.25 & 28.92 & 38.15 & 8.75 & 4.25 & 9.00 & 7.33 & 2.78 \\
\hline DSR 40 & 15.00 & 11.50 & 8.50 & 11.67 & -76.47 & 23.00 & 52.25 & 13.25 & 29.50 & -73.58 & 6.00 & 9.50 & 7.75 & 7.75 & 22.58 \\
\hline DSR 47 & 18.50 & 17.50 & 19.50 & 18.50 & 5.13 & 31.25 & 58.75 & 54.25 & 48.08 & 42.40 & 11.75 & 10.75 & 17.50 & 13.33 & 32.86 \\
\hline Morden & 17.00 & 9.00 & 15.00 & 13.67 & -13.33 & 28.50 & 17.25 & 23.50 & 23.08 & -21.28 & 4.75 & 2.50 & 8.75 & 5.33 & 45.71 \\
\hline RHA 6D-1 & 10.50 & 8.00 & 8.00 & 8.83 & -31.25 & 6.25 & 6.75 & 6.50 & 6.50 & 3.85 & 2.00 & 2.75 & 5.75 & 3.50 & 65.22 \\
\hline RHA 95C-1 & 11.00 & 11.00 & 12.00 & 11.33 & 8.33 & 13.75 & 15.75 & 17.50 & 15.67 & 21.43 & 3.25 & 3.75 & 5.75 & 4.25 & 43.48 \\
\hline Mean & 14.35 & 13.00 & 13.75 & 13.70 & & 23.83 & 25.85 & 31.15 & 26.94 & & 6.68 & 5.90 & 9.73 & 7.43 & \\
\hline \multicolumn{4}{|c|}{ CD at $5 \%$ for Stresses $(S)$} & 1.18 & & & & & 2.27 & & & & & 0.66 & \\
\hline \multicolumn{4}{|c|}{ CD at $5 \%$ for Genotypes G) } & 2.15 & & & & & 4.15 & & & & & 1.20 & \\
\hline \multicolumn{4}{|c|}{ CD at $5 \%$ for Stress $\times$ Genotype } & 3.72 & & & & & 7.19 & & & & & 2.07 & \\
\hline
\end{tabular}

Note: S- Severe stress; MS- Moderate stress; NS- Non-stress/Control/Well watered

Table.4 Mean performance of head dry weight, total dry matter and leaf area under different soil moisture regimes in sunflower

\begin{tabular}{|c|c|c|c|c|c|c|c|c|c|c|c|c|c|c|c|}
\hline \multirow[b]{2}{*}{ Genotypes } & \multicolumn{5}{|c|}{ Head Dry weight $(g)$} & \multicolumn{5}{|c|}{ Total Dry matter (g) } & \multicolumn{5}{|c|}{ Leaf area $(\mathrm{cm} 2)$} \\
\hline & $\mathbf{S}$ & MS & NS & Mean & $\begin{array}{l}\text { Per cent } \\
\text { Reduction }\end{array}$ & $\mathbf{S}$ & MS & NS & Mean & $\begin{array}{l}\text { Per cent } \\
\text { Reduction }\end{array}$ & $\mathbf{S}$ & MS & NS & Mean & $\begin{array}{l}\text { Per cent } \\
\text { Reduction }\end{array}$ \\
\hline DSR 14 & 15.00 & 17.00 & 22.00 & 18.00 & 31.82 & 60.00 & 55.00 & 95.25 & 70.08 & 37.01 & 151.35 & 131.63 & 264.60 & 182.53 & 42.80 \\
\hline DSR 57 & 11.63 & 10.90 & 19.38 & 13.97 & 40.00 & 31.88 & 27.15 & 60.63 & 39.88 & 47.42 & 118.35 & 117.90 & 185.85 & 140.70 & 36.32 \\
\hline DSR 51 & 13.83 & 14.75 & 17.00 & 15.19 & 18.68 & 43.08 & 48.75 & 48.25 & 46.69 & 10.73 & 106.31 & 120.15 & 121.50 & 115.99 & 12.50 \\
\hline DSR 56 & 13.50 & 13.50 & 19.50 & 15.50 & 30.77 & 58.00 & 41.75 & 69.75 & 56.50 & 16.85 & 108.90 & 129.15 & 141.08 & 126.38 & 22.81 \\
\hline DSR 20 & 15.75 & 14.50 & 14.25 & 14.83 & -10.53 & 51.25 & 35.50 & 66.50 & 51.08 & 22.93 & 110.93 & 151.65 & 220.50 & 161.03 & 49.69 \\
\hline DSR 40 & 15.38 & 18.88 & 19.73 & 17.99 & 22.07 & 44.38 & 80.63 & 40.73 & 55.24 & -8.95 & 108.90 & 148.50 & 111.15 & 122.85 & 2.02 \\
\hline DSR 47 & 17.25 & 16.50 & 25.25 & 19.67 & 31.68 & 60.25 & 86.00 & 97.00 & 81.08 & 37.89 & 137.70 & 208.80 & 234.68 & 193.73 & 41.32 \\
\hline Morden & 18.25 & 14.00 & 21.00 & 17.75 & 13.10 & 51.50 & 33.75 & 53.25 & 46.17 & 3.29 & 76.50 & 101.48 & 179.55 & 119.18 & 57.39 \\
\hline RHA 6D-1 & 4.00 & 4.60 & 18.63 & 9.08 & 78.52 & 12.25 & 14.10 & 30.88 & 19.08 & 60.32 & 87.75 & 105.75 & 137.70 & 110.40 & 36.27 \\
\hline RHA 95C-1 & 6.00 & 5.50 & 19.55 & 10.35 & 69.31 & 23.00 & 25.00 & 42.80 & 30.27 & 46.26 & 61.20 & 83.25 & 103.50 & 82.65 & 40.87 \\
\hline Mean & 13.06 & 13.01 & 19.63 & 15.23 & & 43.56 & 44.76 & 60.50 & 49.61 & & 106.79 & 129.83 & 170.01 & 135.54 & \\
\hline \multicolumn{4}{|c|}{ CD at $5 \%$ for Stresses $(\mathrm{S})$} & 0.89 & & & & & 2.56 & & & & & 11.58 & \\
\hline \multicolumn{4}{|c|}{ CD at $5 \%$ for Genotypes G) } & 1.63 & & & & & 4.67 & & & & & 21.14 & \\
\hline \multicolumn{4}{|c|}{ CD at $5 \%$ for Stress $\times$ Genotype } & 2.83 & & & & & 8.10 & & & & & 36.61 & \\
\hline
\end{tabular}

Note: S- Severe stress; MS- Moderate stress; NS- Non-stress/Control/Well watered 
Table.5 Mean performance of head diameter and seed yield per plant of sunflower genotypes as influenced by different soil moisture regimes

\begin{tabular}{|c|c|c|c|c|c|c|c|c|c|c|c|}
\hline \multirow[b]{2}{*}{ Genotypes } & \multicolumn{5}{|c|}{ Head diameter (g) } & \multicolumn{6}{|c|}{ Seed yield/plant $(\mathrm{g})$} \\
\hline & $\mathbf{S}$ & MS & NS & Mean & $\begin{array}{c}\text { Per cent } \\
\text { Reduction }\end{array}$ & $\mathbf{S}$ & MS & NS & Mean & $\begin{array}{c}\text { Per cent } \\
\text { Reduction } \\
\text { by } S \\
\end{array}$ & $\begin{array}{c}\text { Per cent } \\
\text { Reduction } \\
\text { by MS }\end{array}$ \\
\hline DSR 14 & 9.75 & 11.25 & 10.75 & 10.58 & 9.30 & 13.74 & 14.61 & 16.53 & 14.96 & 16.88 & 11.64 \\
\hline DSR 57 & 6.25 & 6.65 & 10.75 & 7.88 & 41.86 & 8.05 & 6.67 & 13.88 & 9.53 & 41.98 & 51.93 \\
\hline DSR 51 & 7.75 & 8.80 & 9.75 & 8.77 & 20.51 & 10.05 & 11.25 & 13.43 & 11.58 & 25.17 & 16.23 \\
\hline DSR 56 & 8.25 & 9.40 & 11.50 & 9.72 & 28.26 & 9.30 & 11.00 & 13.80 & 11.37 & 32.61 & 20.30 \\
\hline DSR 20 & 9.00 & 8.75 & 11.75 & 9.83 & 23.40 & 8.13 & 8.88 & 11.73 & 9.58 & 30.73 & 24.34 \\
\hline DSR 40 & 7.75 & 9.75 & 11.50 & 9.67 & 32.61 & 10.67 & 11.81 & 14.74 & 12.40 & 27.62 & 19.85 \\
\hline DSR 47 & 9.25 & 10.75 & 11.25 & 10.42 & 17.78 & 12.38 & 14.88 & 16.18 & 14.48 & 23.49 & 8.03 \\
\hline Morden & 4.25 & 3.75 & 9.25 & 5.75 & 54.05 & 5.38 & 4.75 & 12.88 & 7.67 & 58.25 & 63.11 \\
\hline RHA 6D-1 & 2.75 & 2.95 & 8.75 & 4.82 & 68.57 & 2.75 & 2.75 & 12.00 & 5.83 & 77.08 & 77.08 \\
\hline RHA 95C-1 & 3.40 & 4.15 & 9.50 & 5.68 & 64.21 & 3.55 & 3.80 & 11.63 & 6.33 & 69.46 & 67.31 \\
\hline Mean & 6.84 & 7.62 & 10.48 & 8.31 & & 8.40 & 9.04 & 13.68 & 10.37 & 38.60 & 33.92 \\
\hline \multicolumn{4}{|c|}{ CD at $5 \%$ for Stresses $(\mathrm{S})$} & 0.68 & & & & & 0.91 & & \\
\hline \multicolumn{4}{|c|}{ CD at $5 \%$ for Genotypes $\mathbf{G}$ ) } & 1.25 & & & & & 1.66 & & \\
\hline \multicolumn{4}{|c|}{ CD at $5 \%$ for Stress $\times$ Genotype } & 2.16 & & & & & 2.87 & & \\
\hline
\end{tabular}

Note: S- Severe stress; MS- Moderate stress; NS- Non-stress/Control/Well watered 
Fig.1 Plant and root morphology of different genotypes of sunflower under severe moisture stress regime

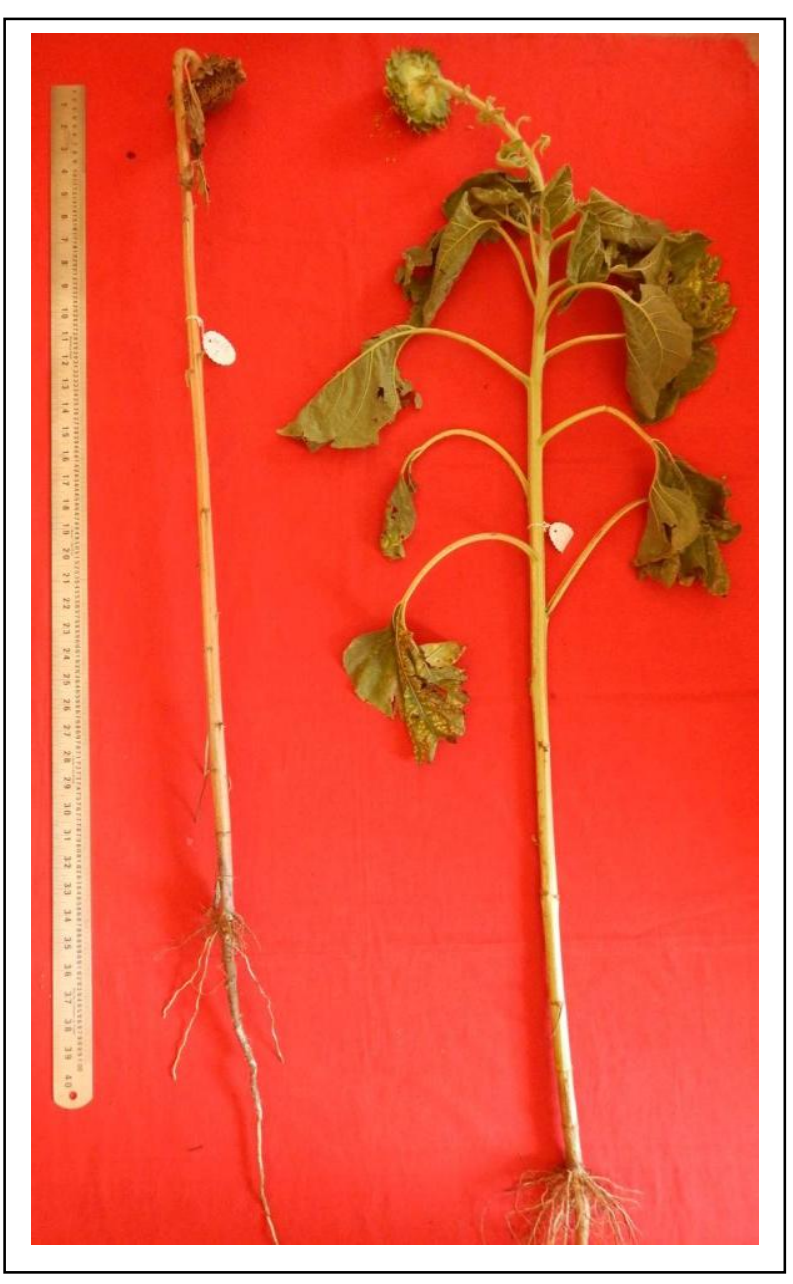

Morden (R1)

DSR 47(R1)

Note: $\mathrm{R} 1$ and $\mathrm{R} 2$ in the parenthesis are replications

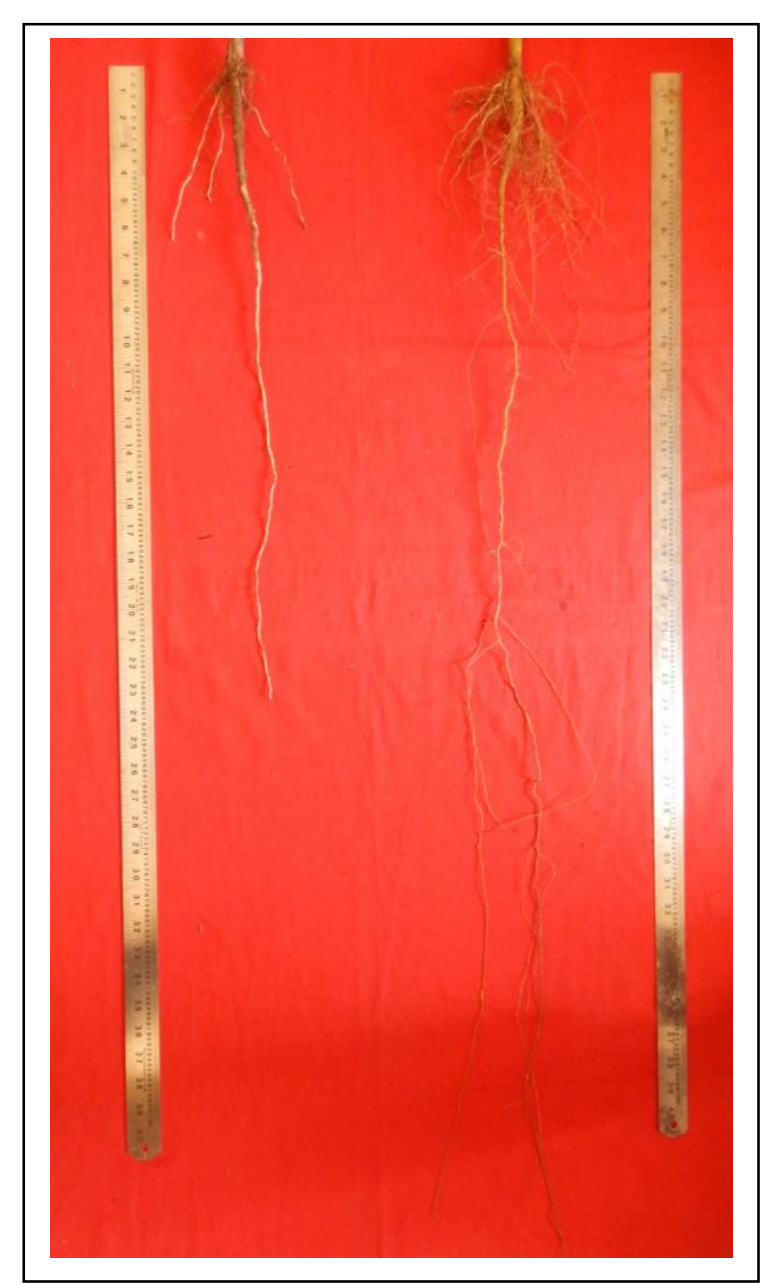

Morden (R1)
DSR 47(R1)

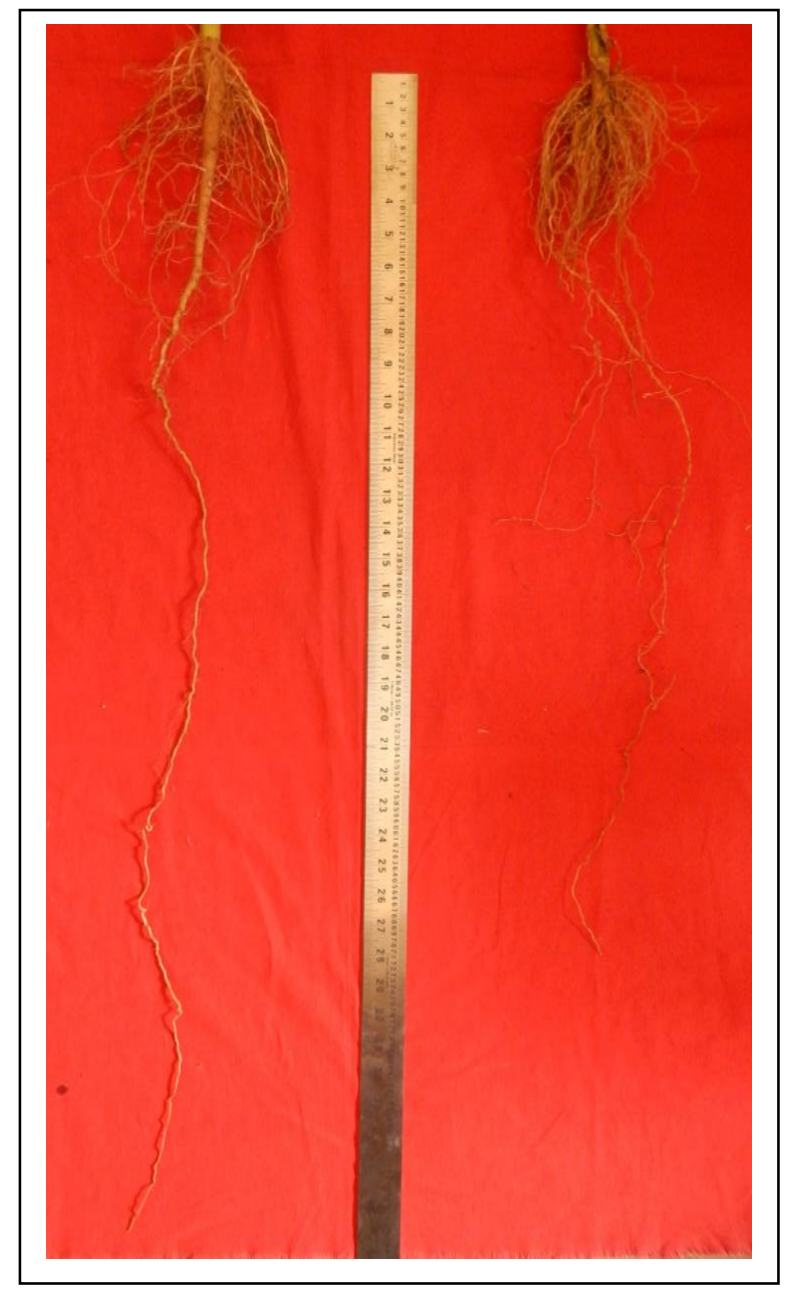

DSR 14 (R2)
DSR 47 (R2) 
Repressing effect on root length, shoot length, root, shoot and total plant fresh and dry weight, leaf area, head diameter and seed yield by moisture stress was reported by several authors (Rauf and Sadaqat, 2007; Manivannan et al., 2007; Rauf and Sadaqat, 2008; Geetha et al., 2012; Oner et al., 2014).

Complex quantitative trait like seed yield is highly influenced by environment. In the present investigation seed yield per plant was significantly differed under different water regimes. It was reduced by $38.60 \%$ and 33.92 $\%$ by the severe and moderate soil moisture stress respectively. The genotypes DSR 14 $(16.88 \%)$ and DSR 47 (23.49 \%) were shown least seed yield reduction under severe moisture stress with mean seed yield of 13.74 $\mathrm{g}$ and $12.38 \mathrm{~g}$ respectively. While under moderate soil moisture stress, the genotype DSR $47(8.03 \%)$ shown least reduction in seed yield per plant followed by DSR 14 $(11.64 \%)$ with mean seed yield of $14.88 \mathrm{~g}$ and $14.61 \mathrm{~g}$, respectively (Table 5). These two genotypes were also found to be superior under well watered condition. Highest seed yield reduction was observed in check genotypes RHA 6D-1, RHA 95C-1 and Morden with seed yield reduction of $77.08 \%$, $69.46 \%$ and $58.25 \%$ respectively under severe soil moisture stress and of $77.08 \%$, $67.31 \%$ and $63.11 \%$ respectively under moderate moisture stress which indicated that these genotypes were shown very least or no recovery in seed yield under moderate stress condition wherein the moisture stress was imposed at critical stage ( $\mathrm{R} 1$ to $\mathrm{R} 2$ stage). The decrease in seed yield might be due to decreased leaf area, head diameter, photosynthetic efficiency by degradation of chlorophyll, lower production and translocation of organic material from source to sink (Amrutha et al., 2007). These results were in accordance with the observations made by other researchers (Bajehbaj, 2011; Tabatabaei et al., 2012; Geetha et al., 2012;
Nagarathna et al., 2012; Ghaffari et al., 2013; Safavi et al., 2015)

Root traits such as root length, root to shoot ratio, root dry weight, leaf area and also total dry matter are found to be significant indicator of drought tolerance in sunflower. Based on the desired root traits the two genotypes DSR 14 and DSR 47 would be utilized for further breeding improvement under drought stress. It was also indicated that, in addition to root traits, developing whole plant strategies by sunflower breeders with considering specifics of genotype as well as genotype $\mathrm{x}$ environment interactions is so key issue in drought resistance breeding.

\section{References}

Ali, Q., Ashraf, M. and Anwar, F., 2009. Physico-chemical attributes of seed oil from drought stress sunflower plants. Grasas y Aceites., 60: 475-481.

Amrutha, R., Muthulaksmi, S., Baby Rani, W., Indira, W. K. and Mareeswari, P. 2007. Alleviation of High Temperature Stress in Sunflower (Helianthus annus L.) By Plant Growth Regulators and Chemicals Research. J. Agric. Biol. Sci., 3 (12): 1658-1662.

Arriola, K. G., Kim, S. C., Huisden, C. M. and Adesogan, A. T., 2012. Staygreen ranking and maturity of corn hybrids: 1 . Effects on dry matter yield, nutritional value, fermentation characteristics, and aerobic stability of silage hybrids in Florida. J. Dairy Sci., 95 (2): 964-974.

Bajehbaj, A. A., 2011. Effects of drought stress and different densities on oil yield and biomass yield of sunflower varieties. African J. Biotechnol., 10 (29): 5608-5613.

Chimenti, C.A., Pearson, J. and Hall, A. J., 2002. Osmotic adjustment and yield maintenance under drought in sunflower. Field Crops Res., 75: 235- 
246.

Comas, L. H., Becker, S. R., Cruz, V. M. V., Byrne, P. F. and Dierig, D. A., 2013. Root traits contributing to plant productivity under drought. Front Plant Science. 4: 442-452.

Eshghi, A., G., Shobeiri, S., Khiavi, M. and Taee, A., 2011. Genetic diversity of sunflower in drought stress condition using root system study in caspian border region of Iran. International symposium on Sunflower genetic resources, Kusadasi, Izmir, Turkey.

Geetha, A., Sivasankar, A., Suresh, J., Lakshmi Prayaga, Saidaih, P. and Anuradha, G., 2012. Genetics of root characteristics in sunflower (Helianthus annuus L.) under contrasting water regimes, J. Oilseeds Res., 29 (1): 109112.

Ghaffari, M., Toorchi, M., Valizadeh, M., and Shakiba, M. R., 2012. Morphophysiological screening of sunflower inbred lines under drought stress condition. Turkish J. Field Crops, 17 (2): 185-190.

Harris, K., Subudhi, P. K., Borrell, A., Jordan, D., Rosenow, D., Nguyen, H., Klein, P., Klein, R. and Mullet, J., 2007, Sorghum stay-green QTL individually reduce post-flowering drought-induced leaf senescence. J Exp Bot., 58 (2): 327-338.

Jordan, D. R., Hunt, C. H., Cruickshank, A. W., Borrell, A. K. and Henzell, R. G., 2012, The relationship between the stay-green trait and grain yield in elite sorghum hybrids grown in a range of environments. Crop Sci., 52 (3): 11531161 .

Manivannan, P., Abdul Jaleel, C., Sankar, B., Kishorekumar, A., Somasundaram, R., Lakshmanan, G. M. A. and Panneerselvam, R., 2007, Growth, biochemical modifications and proline metabolism in Helianthus annuus L. as induced by drought stress. Colloids and
Surfaces B: Biointerfaces, 59: 141-149. Nagarathna, T. K., Shadakshari, Y. G. Ramakrishna Parama, V. R., Jagadish, K. S. and Puttarangaswamy, K. T., 2012, Examination of root characters, isotope discrimination, physiological and morphological traits and their relationship used to identify the drought tolerant sunflower (Helianthus annuus L.) genotypes. Helia, 35 (56): 1-8.

Oner, C., Peter, G. K., Frank, E., Michael, C. F. and Ali, K. M., 2014, Determination of the relationship between water use efficiency, carbon isotope discrimination and proline in sunflower genotypes under drought stress. Aus. J. Crop Sci., 8 (2): 232-242.

Rachidi, F., Kirkham, M. B., Stone, L. R. and Kanemasu, E. T., 1993, Soil water depletion by sunflower and sorghum under rainfed conditions. Agricultural Water Management 24 (1): 49-62.

Rauf, S. and Sadaqat, H. A., 2007, Effects of varied water regimes on root length, dry matter partitioning and endogenous plant growth regulators in sunflower (Helianthus annuus L.). Journal of Plant Interactions, March 2007; 2 (1): 41-51.

Rauf, S. and Sadaqat, H. A., 2008, Identification of physiological traits and genotypes combined to high achene yield in sunflower (Helianthus annuus L.) under contrasting water regimes. Aus. J. Crop Sci., 1 (1): 23-30.

Rong, H., Tang, Y., Zhang, H., Wua, P., Chen, Y., Li, M., Wua, G. and Jiang, H., 2013, The Stay-Green Rice like (SGRL) gene regulates chlorophyll degradation in rice. J Plant Physiol., 170 (15): 1367-1373.

Safavi, S. M., Safavi, A. S. and Safavi, S. A. 2015, Evaluation of drought Tolerance in Sunflower (Helianthus annuus L.) Inbred Lines and Synthetic Varieties under Non Stress and Drought Stress 
Conditions. Biological Forum, 7 (1): 1849-1854.

Satyabrata, M., Hedge, M. R. and Chattopodhay, S. B., 1988, Hand Book of Annual Oilseed Crops. Oxford IBH Pub. Co. (Pvt) Ltd. New Dehli. pp.176.

Skoric, D., 1992, Achievements and future directions of sunflower breeding. Field Crops Research 30: 231-270.

Skoric, D., 2009, Sunflower breeding for resistance to abiotic stresses. Helia, 32(50): 1-16.
Soorninia, F., Toorchi, M., Norouzi, M. and Shakiba, M., 2012, evaluation of sunflower inbred lines under drought Stress. Universal J. Environ. Res. Technol., 2 (1): 70-76.

Tabatabaei, S. A., Rafiee, V., Shakeri, E., And Salmani, M., 2012, Responses of sunflower (Helianthus annuus L.) to deficit irrigation at different growth stages, Intl. J. Agric: Res. Rev., 2 (5): 624-629.

\section{How to cite this article:}

Guruprasad Hiremath and Hajisaheb L. Nadaf. 2017. Assessment of Stay Green Genotypes of Sunflower for Root Traits under Different Soil Moisture Regimes. Int.J.Curr.Microbiol.App.Sci. 6(11): 1156-1166. doi: https://doi.org/10.20546/ijcmas.2017.611.138 